PROCEEDINGS OF THE

AMERICAN MATHEMATICAL SOCIETY

Volume 133, Number 4, Pages 945-951

S 0002-9939(04)07784-6

Article electronically published on November 19, 2004

\title{
SMALL PRIME SOLUTIONS OF QUADRATIC EQUATIONS II
}

\author{
KWOK-KWONG STEPHEN CHOI AND JIANYA LIU \\ (Communicated by David E. Rohrlich)
}

\begin{abstract}
Let $b_{1}, \ldots, b_{5}$ be non-zero integers and $n$ any integer. Suppose that $b_{1}+\cdots+b_{5} \equiv n(\bmod 24)$ and $\left(b_{i}, b_{j}\right)=1$ for $1 \leq i<j \leq 5$. In this paper we prove that (i) if the $b_{j}$ are not all of the same sign, then the above quadratic equation has prime solutions satisfying $p_{j} \ll \sqrt{|n|}+\max \left\{\left|b_{j}\right|\right\}^{25 / 2+\varepsilon}$; and (ii) if all the $b_{j}$ are positive and $n \gg \max \left\{\left|b_{j}\right|\right\}^{26+\varepsilon}$, then the quadratic equation $b_{1} p_{1}^{2}+\cdots+b_{5} p_{5}^{2}=n$ is soluble in primes $p_{j}$. Our previous results are $\max \left\{\left|b_{j}\right|\right\}^{20+\varepsilon}$ and $\max \left\{\left|b_{j}\right|\right\}^{41+\varepsilon}$ in place of $\max \left\{\left|b_{j}\right|\right\}^{25 / 2+\varepsilon}$ and $\max \left\{\left|b_{j}\right|\right\}^{26+\varepsilon}$ above, respectively.
\end{abstract}

For any integer $n$, we consider the quadratic equations in the form

$$
b_{1} p_{1}^{2}+\cdots+b_{5} p_{5}^{2}=n,
$$

where the $p_{j}$ are prime variables and the coefficients $b_{j}$ are non-zero integers. A necessary condition for the solubility of (1) is

$$
b_{1}+\cdots+b_{5} \equiv n \quad(\bmod 24) .
$$

We also suppose

$$
\left(b_{i}, b_{j}\right)=1, \quad 1 \leq i<j \leq 5,
$$

and write $B=\max \left\{2,\left|b_{1}\right|, \ldots,\left|b_{5}\right|\right\}$. The main results in this note are the following two theorems.

Theorem 1. Suppose (21) and (31). If $b_{1}, \ldots, b_{5}$ are not all of the same sign, then (11) has solutions in the primes $p_{j}$ satisfying

$$
p_{j} \ll \sqrt{|n|}+B^{25 / 2+\varepsilon},
$$

where the implied constant depends only on $\varepsilon$.

Theorem 2. Suppose (2) and (3). If $b_{1}, \ldots, b_{5}$ are all positive, then (1) is soluble whenever

$$
n \gg B^{26+\varepsilon},
$$

where the implied constant depends only on $\varepsilon$.

Theorem [2] with $b_{1}=\ldots=b_{5}=1$ is a classical result of Hua [3] in 1938. Theorems 1 and 2 improve our previous results in [1] with the bounds $B^{20+\varepsilon}$ and $B^{41+\varepsilon}$ in the place of $B^{25 / 2+\varepsilon}$ and $B^{26+\varepsilon}$, respectively.

Received by the editors February 3, 2003.

2000 Mathematics Subject Classification. Primary 11P32, 11P05, 11P55.

The first and second authors were supported by the NSERC and the NSF of China (Grant \#10125101), respectively.

(C)2004 American Mathematical Society Reverts to public domain 28 years from publication 
Recently, the second author introduced in [4] an iterative procedure to deal with the enlarged major arcs in the Waring-Goldbach problem which can be used to improve the previous results substantially. In this note, we will demonstrate how to use this iterative procedure to improve our previous results in [1]. Most of the arguments are similar to those in [1] and we therefore only sketch the proof here. We refer the reader to [1] for all the details and only emphasize the main difference between the arguments.

Denote by $r(n)$ the weighted number of solutions of (1), i.e.,

$$
r(n)=\sum_{\substack{n=b_{1} \\ M<\left|b_{j}\right| p_{j}^{2} \leq N}}\left(\log p_{1}\right) \cdots\left(\log p_{5}\right),
$$

where $M=N / 200$. We will investigate $r(n)$ by the circle method. To this end, we set

$$
P=(N / B)^{1 / 5-\varepsilon}, \quad Q=N /\left(P L^{9000}\right), \quad \text { and } \quad L=\log N .
$$

We should remark that the previous choice of $P$ in [1] is $P=(N / B)^{1 / 8-\varepsilon}$. The improvement in our theorems is due to the choice of larger $P$ in (4).

By Dirichlet's lemma on rational approximation, each $\alpha \in[1 / Q, 1+1 / Q]$ may be written in the form

$$
\alpha=a / q+\lambda, \quad|\lambda| \leq 1 /(q Q)
$$

for some integers $a, q$ with $1 \leq a \leq q \leq Q$ and $(a, q)=1$. We denote by $\mathfrak{M}(a, q)$ the set of $\alpha$ satisfying (5), and define the major arcs $\mathfrak{M}$ and the minor arcs $\mathfrak{m}$ as follows:

$$
\mathfrak{M}=\bigcup_{q \leq P} \bigcup_{\substack{a=1 \\(a, q)=1}}^{q} \mathfrak{M}(a, q), \quad \mathfrak{m}=\left[\frac{1}{Q}, 1+\frac{1}{Q}\right] \backslash \mathfrak{M} .
$$

It follows from $2 P \leq Q$ that the major arcs $\mathfrak{M}(a, q)$ are mutually disjoint. Let

$$
S_{j}(\alpha)=\sum_{M<\left|b_{j}\right| p^{2} \leq N}(\log p) e\left(b_{j} p^{2} \alpha\right),
$$

where $e(x):=e^{2 \pi i x}$. Then we have

$$
r(n)=\int_{0}^{1} S_{1}(\alpha) \cdots S_{5}(\alpha) e(-n \alpha) d \alpha=\int_{\mathfrak{M}}+\int_{\mathfrak{m}} .
$$

For $\chi \bmod q$, we define

$$
C(\chi, a)=\sum_{h=1}^{q} \bar{\chi}(h) e\left(\frac{a h^{2}}{q}\right), \quad C(q, a)=C\left(\chi^{0}, a\right) .
$$

Here $\chi^{0}$ is the principal character $\bmod q$. If $\chi_{1}, \ldots, \chi_{5}$ are characters $\bmod q$, then we write

$$
B\left(n, q, \chi_{1}, \ldots, \chi_{5}\right)=\sum_{\substack{a=1 \\(a, q)=1}}^{q} e\left(-\frac{a n}{q}\right) C\left(\chi_{1}, b_{1} a\right) \cdots C\left(\chi_{5}, b_{5} a\right),
$$

and

$$
\mathfrak{S}(n, x)=\sum_{q \leq x} \frac{B\left(n, q, \chi^{0}, \ldots, \chi^{0}\right)}{\varphi^{5}(q)}
$$


where $\varphi(q)$ is the Euler totient function. The integral on the major arcs $\mathfrak{M}$ causes the main difficulty, which is solved by the following.

Theorem 3. Assume (3). Let $\mathfrak{M}$ be as in (6) with $P$ and $Q$ determined by (4). If $N \geq P^{5+\varepsilon} B$, then we have

$$
\int_{\mathfrak{M}} S_{1}(\alpha) \cdots S_{5}(\alpha) e(-n \alpha) d \alpha=\frac{1}{32} \mathfrak{S}(n, P) \mathfrak{I}(n)+O\left(\frac{N^{3 / 2}}{\left|b_{1} \cdots b_{5}\right|^{1 / 2} L}\right),
$$

where $\mathfrak{S}(n, P)$ is defined in (8) and

$$
\Im(n):=\sum_{\substack{b_{1} m_{1}+\cdots+b_{5} m_{5}=n \\ M<\left|b_{j}\right| m_{j} \leq N}}\left(m_{1} \cdots m_{5}\right)^{-1 / 2} .
$$

As shown in [1, the integral on $\mathfrak{m}$ satisfies

$$
\left|\int_{\mathfrak{m}}\right| \ll \frac{N^{3 / 2+\varepsilon}}{\left|b_{1} \cdots b_{5}\right|^{1 / 4} P^{1 / 4}} .
$$

The contribution from the major arcs can be handled by Theorem 3 , which together with (7) and (9) gives

$$
r(n)=\frac{1}{32} \mathfrak{S}(n, P) \mathfrak{I}(n)+O\left(\frac{N^{3 / 2}}{\left|b_{1} \cdots b_{5}\right|^{1 / 2} L}+\frac{N^{3 / 2+\varepsilon}}{\left|b_{1} \cdots b_{5}\right|^{1 / 4} P^{1 / 4}}\right) .
$$

The lower bounds for $\mathfrak{S}(n, P)$ and $\mathfrak{I}(n)$ were estimated in [1]. The following are Lemmas 2.1 and 2.2 in [1].

Lemma 4. Assuming (2), we have $\mathfrak{S}(n, P) \gg(\log \log B)^{-c_{1}}$ for some constant $c_{1}>0$.

Lemma 5. Suppose (3) and either (i) the $b_{j}$ 's are not all of the same sign and $N \geq 10|n|$; or (ii) all the $b_{j}$ 's are positive and $n=N$. Then we have

$$
\Im(n) \asymp \frac{N^{3 / 2}}{\left|b_{1} \cdots b_{5}\right|^{1 / 2}} .
$$

Now assume either condition (i) or (ii) in Lemma 5 Applying Lemmas 4 and 5 to the above formula, we conclude that

$$
r(n) \gg\left|b_{1} \cdots b_{5}\right|^{-1 / 2} N^{3 / 2}(\log \log B)^{-c_{1}}
$$

provided that $P \gg N^{\varepsilon}\left|b_{1} \cdots b_{5}\right|$, or equivalently $N \gg B^{1+\varepsilon}\left|b_{1} \cdots b_{5}\right|^{5}$. This proves Theorems 1 and 2 .

Therefore, it remains to prove Theorem 3 .

For $j=1, \ldots, 5$, set

$$
V_{j}(\lambda)=\sum_{M<\left|b_{j}\right| m^{2} \leq N} e\left(b_{j} m^{2} \lambda\right)
$$

and

$$
W_{j}(\chi, \lambda)=\sum_{M<\left|b_{j}\right| p^{2} \leq N}(\log p) \chi(p) e\left(b_{j} p^{2} \lambda\right)-\delta_{\chi} \sum_{M<\left|b_{j}\right| m^{2} \leq N} e\left(b_{j} m^{2} \lambda\right),
$$

where $\delta_{\chi}=1$ or 0 according to whether $\chi$ is principal or not. We can rewrite the exponential sum $S_{j}(\alpha)$ as (see for example [2], §26, (2))

$$
S_{j}\left(\frac{h}{q}+\lambda\right)=\frac{C\left(q, b_{j} h\right)}{\varphi(q)} V_{j}(\lambda)+\frac{1}{\varphi(q)} \sum_{\chi \bmod q} C\left(\chi, b_{j} h\right) W_{j}(\chi, \lambda)=: T_{j}+U_{j},
$$


say. Thus,

$$
\int_{\mathfrak{M}} S_{1}(\alpha) \cdots S_{5}(\alpha) e(-n \alpha) d \alpha=I_{0}+\cdots+I_{5},
$$

where $I_{\nu}$ denotes the contribution from those products with $\nu$ pieces of $U_{j}$ and $5-\nu$ pieces of $T_{j}$, i.e.,

$$
I_{\nu}=\sum_{q \leq P} \sum_{\substack{a=1 \\(a, q)=1}}^{q} e\left(-\frac{a n}{q}\right) \int_{-1 /(q Q)}^{1 /(q Q)}\left(U_{1} \cdots U_{\nu} T_{\nu+1} \cdots T_{5}+\text { s.t. }\right) e(-n \lambda) d \lambda,
$$

where "s.t." means similar terms. For example, " $A_{1} B_{2} C_{3} D_{4} E_{5}+$ s.t." means the sum of all possible terms $A_{\alpha} B_{\beta} C_{\gamma} D_{\delta} E_{\iota}$ with $(\alpha, \ldots, \iota)$ being any permutation of $(1, \ldots, 5)$.

We will prove that $I_{0}$ gives the main term and $I_{1}, \ldots, I_{5}$ the error term. The estimation of $I_{0}$ is the same as that in [1] and we have

$$
I_{0}=\frac{1}{32} \mathfrak{S}(n, P) \mathfrak{I}(n)+O\left(\frac{N^{3 / 2}}{\left|b_{1} \cdots b_{5}\right|^{1 / 2} L}\right) .
$$

It remains to show that $\left|I_{i}\right| \ll N^{3 / 2}\left|b_{1} \cdots b_{5}\right|^{-1 / 2} L^{-1}$ for $1 \leq i \leq 5$. To this end, we define, for any $g \geq 1$

$$
J_{j}(g)=\sum_{r \leq P}[g, r]^{-1+\varepsilon} \sum_{\chi \bmod r}^{*} \max _{|\lambda| \leq 1 /(r Q)}\left|W_{j}(\chi, \lambda)\right|
$$

and

$$
K_{j}(g)=\sum_{r \leq P}[g, r]^{-1+\varepsilon} \sum_{\chi \bmod r} *\left(\int_{-1 /(r Q)}^{1 /(r Q)}\left|W_{j}(\chi, \lambda)\right|^{2} d \lambda\right)^{1 / 2},
$$

where $\sum_{\chi \bmod r}{ }^{*}$ is over all the primitive characters modulo $r$ and $[g, r]$ is the least common multiple of $g$ and $r$.

Our Theorem 3 depends on the following three main lemmas.

Lemma 6. For $P, Q$ satisfying (4), we have

$$
J_{j}(g) \ll g^{-1+2 \varepsilon} N^{1 / 2}\left|b_{j}\right|^{-1 / 2} L^{c}
$$

for some constant $c>0$.

Lemma 7. Let $P, Q$ satisfy (4). For $g=1$, Lemma 6 can be improved to

$$
J_{j}(1) \ll N^{1 / 2}\left|b_{j}\right|^{-1 / 2} L^{-A},
$$

where $A>0$ is arbitrary.

Lemma 8. For $P, Q$ satisfying (4), we have

$$
K_{j}(g) \ll g^{-1+2 \varepsilon}\left|b_{j}\right|^{-1 / 2} L^{c}
$$

for some constant $c>0$.

We omit the proof of Lemmas [6], since they can be proved by combining the corresponding arguments in [4] and [1]. In fact, Lemmas 6] 8] with $b_{j}=1$ can be established in exactly the same way as Lemmas 3.1-3.3 of [4], which depend on Lemma 2.1 of [4], a hybrid estimate for Dirichlet polynomials. Lemmas 6$]$ are essential in our iterative argument below; another application of the iterative method appears in [5]. 
For example, following the same proof of Lemma 3.1 of [4], one can show that our Lemma 6 is a consequence of the following two estimates: For $R \leq P$ and $0<T_{1} \leq T_{0}$, we have

$$
\sum_{r \sim R}[g, r]^{-1+\varepsilon} \sum_{\chi \bmod r}^{*} \int_{T_{1}}^{2 T_{1}}\left|F\left(\frac{1}{2}+i t, \chi\right)\right| d t \ll g^{-1+2 \varepsilon} N_{j}^{1 / 4}\left(T_{1}+1\right)^{1 / 2} L^{c},
$$

while for $R \leq P$ and $T_{0}<T_{2} \leq T$, we have

$$
\sum_{r \sim R}[g, r]^{-1+\varepsilon} \sum_{\chi \bmod r}^{*} \int_{T_{2}}^{2 T_{2}}\left|F\left(\frac{1}{2}+i t, \chi\right)\right| d t \ll g^{-1+2 \varepsilon} N_{j}^{1 / 4} T_{2} L^{c} .
$$

Here $T_{0}=8 \pi N /(R Q), N_{j}=N /\left|b_{j}\right|$, and $F(s, \chi)$ is as in $\S 2$ of [4] with $X=N_{j}^{1 / 2}$ and $Y=\left(N_{j} / 200\right)^{1 / 2}$. To show (11), we note that $[g, r](g, r)=g r$. Then the left-hand side of (11) is

$$
\ll g^{-1+\varepsilon} \sum_{\substack{d \mid g \\ d \leq R}}\left(\frac{R}{d}\right)^{-1+\varepsilon} \sum_{\substack{r \sim R \\ d \mid r}} \sum_{\chi \bmod r}^{*} \int_{T_{1}}^{2 T_{1}}\left|F\left(\frac{1}{2}+i t, \chi\right)\right| d t .
$$

Let $\tau(g)$ be the divisor function. By Lemma 2.1 in [4, the above quantity can be estimated as

$$
\begin{aligned}
& \ll g^{-1+\varepsilon} \sum_{\substack{d \mid g \\
d \leq R}}\left(\frac{R}{d}\right)^{-1+\varepsilon}\left(\frac{R^{2}}{d} T_{1}+\frac{R}{d^{1 / 2}} T_{1}^{1 / 2} N_{j}^{3 / 20}+N_{j}^{1 / 4}\right) L^{c} \\
& \ll g^{-1+\varepsilon} \tau(g)\left(R^{1+\varepsilon} T_{1}+R^{1 / 2+\varepsilon} T_{1}^{1 / 2} N_{j}^{3 / 20}+N_{j}^{1 / 4}\right) L^{c} \\
& \ll g^{-1+2 \varepsilon} N_{j}^{1 / 4}\left(T_{1}+1\right)^{1 / 2} L^{c},
\end{aligned}
$$

provided that $R \leq N_{j}^{1 / 5-\varepsilon}$. This requirement is necessary, since otherwise $R^{1 / 2+\varepsilon} T_{1}^{1 / 2} N_{j}^{3 / 20}$ cannot be bounded from above by $N_{j}^{1 / 4}\left(T_{1}+1\right)^{1 / 2}$. This establishes (11). Similarly we can prove (12). Therefore $P=(N / B)^{1 / 5-\varepsilon}$ in (41) is the optimal choice. The general assertions in Lemmas 6$] 8$ can be obtained as in Lemmas $4.1,4.2$, and 5.1 of [1].

We demonstrate by estimating $I_{5}$ here, and the treatment of the other $I_{i}$ are similar. We first reduce the characters in $I_{5}$ into primitive characters, to get

$$
\begin{aligned}
\left|I_{5}\right|= & \mid \sum_{q \leq P} \sum_{\chi_{1} \bmod q} \ldots \sum_{\chi_{5} \bmod q} \frac{B\left(n, q, \chi_{1}, \ldots, \chi_{5}\right)}{\varphi^{5}(q)} \\
& \times \int_{-1 /(q Q)}^{1 /(q Q)} W_{1}\left(\chi_{1}, \lambda\right) \cdots W_{5}\left(\chi_{5}, \lambda\right) e(-n \lambda) d \lambda \mid \\
\leq & \sum_{r_{1} \leq P} \cdots \sum_{r_{5} \leq P} \sum_{\chi_{1} \bmod r_{1}}^{*} \ldots \sum_{\chi_{5} \bmod r_{5}}^{*} \sum_{q \leq P} \frac{\left|B\left(n, q, \chi_{1} \chi^{0}, \ldots, \chi_{5} \chi^{0}\right)\right|}{\varphi^{5}(q)} \\
& \times \int_{-1 /(q Q)}^{1 /(q Q)}\left|W_{1}\left(\chi_{1} \chi^{0}, \lambda\right)\right| \cdots\left|W_{5}\left(\chi_{5} \chi^{0}, \lambda\right)\right| d \lambda,
\end{aligned}
$$

where $r_{0}=\left[r_{1}, \ldots, r_{5}\right]$. For $q \leq P$ and $M<\left|b_{j}\right| p^{2} \leq N$, we have $(q, p)=1$. Using this and (10), we have $W_{j}\left(\chi_{j} \chi^{0}, \lambda\right)=W_{j}\left(\chi_{j}, \lambda\right)$ for the primitive characters $\chi_{j}$ 
above. Consequently by Lemma 3.1 in [1], we obtain

$$
\begin{aligned}
\left|I_{5}\right| \leq & \sum_{r_{1} \leq P} \cdots \sum_{r_{5} \leq P} \sum_{\chi_{1} \bmod r_{1}}{ }^{*} \cdots \sum_{\chi_{5} \bmod r_{5}} * \int_{-1 /\left(r_{0} Q\right)}^{1 /\left(r_{0} Q\right)}\left|W_{1}\left(\chi_{1}, \lambda\right)\right| \cdots\left|W_{5}\left(\chi_{5}, \lambda\right)\right| d \lambda \\
& \times \sum_{\substack{q \leq P \\
r_{0} \mid q}} \frac{\left|B\left(n, q, \chi_{1} \chi^{0}, \ldots, \chi_{5} \chi^{0}\right)\right|}{\varphi^{5}(q)} \\
\ll & L^{c_{2}} \sum_{r_{1} \leq P} \cdots \sum_{r_{5} \leq P} r_{0}^{-1+\varepsilon} \sum_{\chi_{1} \bmod r_{1}}{ }^{*} \cdots \sum_{\chi_{5} \bmod r_{5}}{ }^{*} \int_{-1 /\left(r_{0} Q\right)}^{1 /\left(r_{0} Q\right)}\left|W_{1}\left(\chi_{1}, \lambda\right)\right| \cdots\left|W_{5}\left(\chi_{5}, \lambda\right)\right| d \lambda .
\end{aligned}
$$

The previous estimate of $I_{5}$ used the trivial inequality $r_{0}^{-1+\varepsilon} \leq r_{1}^{-1 / 5+\varepsilon} \cdots r_{5}^{-1 / 5+\varepsilon}$. Instead of using this inequality which is responsible for a weaker result, we employ an iterative argument introduced in [4] to bound the above sums over $r_{1}, r_{2}, r_{3}, r_{4}, r_{5}$ consecutively. By Cauchy's inequality, we get

$$
\begin{aligned}
\left|I_{5}\right| \ll & L^{c_{2}} \sum_{r_{1} \leq P} \sum_{\chi_{1} \bmod r_{1}}^{*} \max _{|\lambda| \leq 1 /\left(r_{1} Q\right)}\left|W_{1}\left(\chi_{1}, \lambda\right)\right| \\
& \times \cdots \times \sum_{r_{3} \leq P \chi_{3}} \sum_{\bmod r_{3}}^{*} \max _{|\lambda| \leq 1 /\left(r_{3} Q\right)}\left|W_{3}\left(\chi_{3}, \lambda\right)\right| \\
& \times \sum_{r_{4} \leq P} \sum_{\chi_{4} \bmod r_{4}} *\left(\int_{-1 /\left(r_{4} Q\right)}^{1 /\left(r_{4} Q\right)}\left|W_{4}\left(\chi_{4}, \lambda\right)\right|^{2} d \lambda\right)^{1 / 2} \\
& \times \sum_{r_{5} \leq P} r_{0}^{-1+\varepsilon} \sum_{\chi_{5} \bmod r_{5}} *\left(\int_{-1 /\left(r_{5} Q\right)}^{1 /\left(r_{5} Q\right)}\left|W_{5}\left(\chi_{5}, \lambda\right)\right|^{2} d \lambda\right)^{1 / 2}
\end{aligned}
$$

The summation over $r_{5}$ on the last line is $K_{5}\left(\left[r_{1}, r_{2}, r_{3}, r_{4}\right]\right)$. Therefore, by Lemma 8

$$
K_{5}\left(\left[r_{1}, r_{2}, r_{3}, r_{4}\right]\right) \ll\left[r_{1}, r_{2}, r_{3}, r_{4}\right]^{-1+2 \varepsilon}\left|b_{5}\right|^{-1 / 2} L^{c_{3}} .
$$

The contribution of the above quantity to the summation over $r_{4}$ in $(13)$ is, by Lemma 8 again,

$$
\begin{aligned}
& \ll\left|b_{5}\right|^{-1 / 2} L^{c_{3}} \sum_{r_{4} \leq P}\left[r_{1}, r_{2}, r_{3}, r_{4}\right]^{-1+2 \varepsilon} \sum_{\chi_{4} \bmod r_{4}} *\left(\int_{-1 /\left(r_{4} Q\right)}^{1 /\left(r_{4} Q\right)}\left|W_{4}\left(\chi_{4}, \lambda\right)\right|^{2} d \lambda\right)^{1 / 2} \\
& =\left|b_{5}\right|^{-1 / 2} L^{c_{3}} K_{4}\left(\left[r_{1}, r_{2}, r_{3}\right]\right) \\
& \ll\left[r_{1}, r_{2}, r_{3}\right]^{-1+4 \varepsilon}\left|b_{4} b_{5}\right|^{-1 / 2} L^{c_{4}} .
\end{aligned}
$$

Using Lemma [6, we can compute the contribution of the above quantity to the sum over $r_{3}$ in (13) as follows:

$$
\begin{aligned}
& \ll\left|b_{4} b_{5}\right|^{-1 / 2} L^{c_{4}} \sum_{r_{3} \leq P}\left[r_{1}, r_{2}, r_{3}\right]^{-1+4 \varepsilon} \sum_{\chi_{3} \bmod r_{3}}{ }^{*} \max _{|\lambda| \leq 1 /\left(r_{3} Q\right)}\left|W_{3}\left(\chi_{3}, \lambda\right)\right| \\
& =\left|b_{4} b_{5}\right|^{-1 / 2} L^{c_{4}} J_{3}\left(\left[r_{1}, r_{2}\right]\right) \\
& \ll\left|b_{3} b_{4} b_{5}\right|^{-1 / 2}\left[r_{1}, r_{2}\right]^{-1+8 \varepsilon} N^{1 / 2} L^{c_{5}} .
\end{aligned}
$$


Inserting this into (13) and applying Lemma 7 we have

$$
\begin{aligned}
I_{5} & \ll N^{1 / 2}\left|b_{3} b_{4} b_{5}\right|^{-1 / 2} L^{c_{5}} \sum_{r_{1} \leq P} \sum_{\chi_{1} \bmod r_{1}}^{*} \max _{|\lambda| \leq 1 /\left(r_{1} Q\right)}\left|W_{1}\left(\chi_{1}, \lambda\right)\right| J_{2}\left(r_{1}\right) \\
& \ll N\left|b_{2} b_{3} b_{4} b_{5}\right|^{-1 / 2} L^{c_{6}} J_{1}(1) \\
& \ll N^{3 / 2}\left|b_{1} b_{2} b_{3} b_{4} b_{5}\right|^{-1 / 2} L^{-A}
\end{aligned}
$$

for arbitrary $A>0$ by applying Lemma 6 to $J_{2}$ and Lemma 7 to $J_{1}$. Similarly we have $\left|I_{4}\right|, \ldots,\left|I_{1}\right| \ll N^{3 / 2}\left|b_{1} b_{2} b_{3} b_{4} b_{5}\right|^{-1 / 2} L^{-A}$. This completes our proof.

\section{REFERENCES}

[1] K. K. Choi and J. Y. Liu, Small prime solutions of quadratic equations, Canad. J. Math. 54(2002), 71-91. MR,1880960 (2002k:11175)

[2] H. Davenport, Multiplicative Number Theory, 2nd ed., Springer, Berlin, 1980. MR0606931 (82m:10001)

[3] L. K. Hua, Some results in the additive prime number theory, Quart. J. Math. (Oxford) 9(1938), 68-80.

[4] J. Y. Liu, On Lagrange's theorem with prime variables, Quart. J. Math. (Oxford) 54(2003), 453-462. MR2031178

[5] J. Y. Liu and T. Zhan, An iterative method in the Waring-Goldbach problem, to appear.

Department of Mathematics, Simon Fraser University, Burnaby, British Columbia, CANADA V5A 1S6

E-mail address: kkchoi@cecm.sfu.ca

Department of Mathematics, Shandong University, Jinan, Shandong 250100, People's Republic of China

E-mail address: jyliu@sdu.edu.cn 